\title{
Simulating surfactant spreading: Influence of a physically motivated equation of state $\dagger$
}

\author{
DINA SINCLAIR ${ }^{1}$, RACHEL LEVY ${ }^{1}$ and KAREN E. DANIELS ${ }^{2}$ \\ ${ }^{1}$ Mathematics Department, Harvey Mudd College, Claremont, CA, USA \\ emails:dsinclair@g.hmc.edu,levy@g.hmc.edu \\ ${ }^{2}$ Department of Physics, North Carolina State University, Raleigh, NC USA \\ email: kdaniel@ncsu.edu
}

(Received 16 February 2016; revised 14 January 2017; accepted 24 January 2017;

first published online 9 March 2017)

\begin{abstract}
In this paper, we present numerical simulations that demonstrate the effect of the particular choice of the equation of state (EoS) relating the surfactant concentration to the surface tension in surfactant-driven thin liquid films. Previous choices of the model EoS have been an ad-hoc decreasing function. Here, we instead propose an empirically motivated EoS; this provides a route to resolve some discrepancies and raises new issues to be pursued in future experiments. In addition, we test the influence of the choice of initial conditions and values for the non-dimensional groups. We demonstrate that the choice of EoS improves the agreement in surfactant distribution morphology between simulations and experiments, and influences the dynamics of the simulations. Because an empirically motivated EoS has regions with distinct gradients, future mathematical models may be improved by considering more than one timescale. We observe that the non-dimensional number controlling the relative importance of gravitational versus capillary forces has a larger influence on the dynamics than the other non-dimensional groups, but is nonetheless not a likely cause of discrepancy between simulations and experiments. Finally, we observe that the experimental approach using a ring to contain the surfactant could affect the surfactant and fluid dynamics if it disrupts the intended initial surfactant distribution. However, the fluid meniscus itself does not significantly affect the dynamics.
\end{abstract}

Key words: AMS classifications: 35Q35 PDEs in connection with fluid mechanics, 76A20 thin fluid films, 76M12 finite volume methods, 76B45 capillarity (surface tension)

\section{Introduction}

Chemicals that lower the surface tension of a fluid are known as surfactants (shorthand for surface active agents). The ability to predict and control the rate and extent to which surfactants spread over the surface of a fluid is important to improve their use in many applications. For example, they are present in healthy lungs to enable breathing, and are also used in industrial applications as stabilizers and dispersants [24]. In human lungs, issues such as airway closure and reopening [31] and the dynamics of mucus in the

$\dagger$ This work was funded by NSF grant DMS-FRG \#096815 (RL and KED), Howard Hughes Medical Institute Undergraduate Science Education Program Award \#52006301 (RL), and Research Corporation Cottrell Scholar Award \#19788 (RL). 

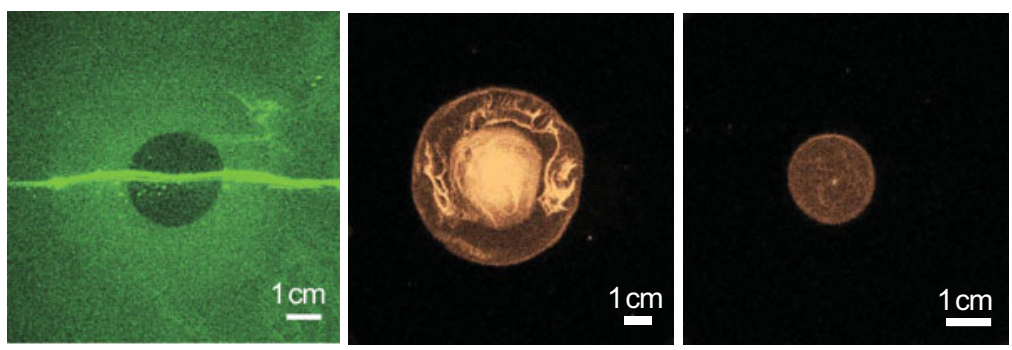

FiguRE 1. Images of fluorescently tagged surfactant spreading from experiments $[39,40]$ viewed from above. Brighter regions have a larger concentration of surfactant. Left: inward spreading below $\tilde{\Gamma}_{c}$ with laser line to show fluid profile with capillary ridges. Middle: outward spreading above $\tilde{\Gamma}_{c}$ with central "reservoir" region of high surfactant concentration. Right: outward spreading below $\tilde{\Gamma}_{c}$ with more uniform surfactant concentration. Figure adapted from $[39,40]$.

airway system $[6,8,16,26]$ are known to be tied to the presence of surfactants. Biomedical engineers and applied mathematicians studying the liquid lining of the lungs of premature infants proposed a compelling model starting with a well-known thin film equation and coupling the film to the surfactant through surface stress $[14,15]$. The stress on the fluid is created by concentration gradients in the layer of insoluble surfactant (and thus a surface tension gradient in the fluid). This, in turn, induces transport of that surfactant on the surface as the fluid moves [21,22].

The model takes the form of two fourth-order non-linear parabolic-hyperbolic partial differential equations. Mathematical interest in these model equations have led to several fruitful approaches to solutions such as asymptotics, similarity solutions, and numerical simulations $[2,11,20,27]$. The solutions provide predictions of spreading behaviour including a spreading timescale as well as fluid and surfactant spatial distributions over time. To test the model, experiments by physicists [12,39] have provided new measurements of the motion of both the surfactant molecules and the underlying fluid, directly testing the validity of the model. While this has led to the identification of some limitations in the model $[39,40]$, the new data also suggest possible improvements.

This paper introduces evidence from the experiments back into the model and suggests avenues for future experiments. For simplicity, we focus on two simple spreading geometries which we will call outward spreading [12] and inward spreading [39]. In both sets of experiments, the system starts with a uniform, millimetric film of glycerol placed on a silicon wafer within a large cylindrical container. A much smaller retaining ring is placed at the surface of the fluid, in the centre of the container. In the outward spreading experiments, the surfactant is placed inside the retaining ring so that it spreads outward once the ring is lifted. In the inward spreading experiments, the surfactant is placed outside the ring and spreads inwards. In simulations, these two cases will be implemented through analogous initial conditions.

In both cases, it is not just the surfactant layer that moves: the glycerol itself is advected by the surfactant, pulled towards regions with less surfactant (and consequently higher surface tension). In order to track the surface distribution of the surfactant molecules, experiments use the fluorescently tagged lipid NBD-PC [1]. Simultaneous measurements of the thickness of the glycerol layer (via an oblique laser line) and the surfactant 
concentration (fluorophores excited by LEDs) are possible; details about experimental procedures are provided in [12,39]. Sample fluorescence images are shown in Figure 1, with the laser visible in the left image.

Importantly, the simulations presented in this paper provide evidence that using an empirically based equation of state (EoS) improves agreement between solutions of the model and the shape of the surfactant distribution in experimental observations. In general, the simulation dynamics of the surfactant profile morphology differ above versus below the critical surfactant concentration $\tilde{\Gamma}_{c}$. This effect has also been observed in outward spreading experiments as seen in the middle and right images of Figure 1. The simulations confirm that unlike the surfactant distribution, the shape of the fluid surface is only weakly dependent on the choice of EoS, explaining why there has previously been reasonable agreement in the fluid profile between models and experiments even without an empirically based EoS.

The use of an empirically based EoS may also provide new insight into the previous lack of agreement in the timescale of the simulations and experiments. First, because the empirical equation of state (EEoS) has three distinct regimes (corresponding to material phases), a single timescale and spreading parameter may not be adequate. Second, although an initial fluid meniscus does not seem to have a major effect on dynamics, a surfactant meniscus could be created by experimental conditions and impact the spreading rate. Third, horizontal shifts in the equations of state (including misidentification of the critical monolayer concentration or the choice of an unrealistic model), can have significant impacts on both the spatial and temporal dynamics. In contrast, the choice of non-dimensional parameters seems unlikely to be the cause of lack of agreement. The simulation results will therefore help guide future modelling efforts, as well as motivate new experimental explorations.

\section{Mathematical model}

A set of equations first proposed in [15] has often been used to model an insoluble surfactant spreading on a thin viscous fluid film $[5,12,34,44,45]$. The equation for the shape of the upper fluid surface (fluid height) $h(x, y, t)$ is based on the well-accepted lubrication approximation and thin film equation, which models the flow of a thin viscous fluid. The height equation additionally incorporates the assumptions that the uncontaminated fluid has small variations in surface tension (modelled as constant) and that surfactant gradients induce surface stress through the tangential boundary condition. The second equation for surfactant concentration $\Gamma(x, y, t)$ assumes that the fluid advects the surfactant by matching the velocities at the fluid-surfactant interface. An ad-hoc term incorporates surfactant diffusion; an alternative derivation based on free-energy considerations has also been proposed [32].

The resulting system of PDE in its common non-dimensional form is

$$
\begin{gathered}
h_{t}+\nabla \cdot\left(\frac{1}{2} h^{2} \nabla \sigma\right)=\beta \nabla \cdot\left(\frac{1}{3} h^{3} \nabla h\right)-\kappa \nabla \cdot\left(\frac{1}{3} h^{3} \nabla \nabla^{2} h\right), \\
\Gamma_{t}+\nabla \cdot(h \Gamma \nabla \sigma)=\beta \nabla \cdot\left(\frac{1}{2} h^{2} \Gamma \nabla h\right)-\kappa \nabla \cdot\left(\frac{1}{2} h^{2} \Gamma \nabla \nabla^{2} h\right)+\delta \nabla^{2} \Gamma,
\end{gathered}
$$


where $h(x, y, t)$ is the fluid height, $\Gamma(x, y, t)$ is the surfactant concentration and $\sigma(\Gamma)$ is the EoS, a constitutive relationship between surfactant concentration and surface tension. The gradient operator is two-dimensional $\left(\nabla=\partial_{x} \hat{x}+\partial_{y} \hat{y}\right)$. We define $r=\sqrt{x^{2}+y^{2}}$ for use in some expressions below. For detailed derivations of this well-studied model, please see $[25,33]$.

\subsection{Non-dimensionalization parameters}

The non-dimensionalization in the above equations is standard: $x=\tilde{x} / R, y=\tilde{y} / R$, $h=\tilde{h} / H$ and $\Gamma=\tilde{\Gamma} / \tilde{\Gamma}_{c}$, where tildes indicate dimensional variables (to contrast with their non-dimensional analogues), $R$ and $H$ are the lateral and vertical length scales, respectively, and $\tilde{\Gamma}_{c}$ is the critical monolayer concentration [23,35]. Time is non-dimensionalized as in [39], motivated by [14]: $\tilde{t}=\left(\frac{\mu R^{2}}{S H}\right) t$, where $\mu$ is the dynamic viscosity and $S \equiv \sigma_{\max }-\sigma_{\min }$ is the spreading parameter set by the $\max / \mathrm{min}$ values of the surface tension. The three non-dimensional parameters in the model are $\beta \equiv \frac{\rho g H^{2}}{S}$ (the ratio of gravity to capillary forces, based on fluid density $\rho$ and gravitational acceleration $g$ ), $\kappa \equiv \frac{\sigma_{\max } H^{2}}{S R^{2}}$ (the ratio of total to relative capillarity scaled by small parameter $H / R$ ) and $\delta \equiv \frac{\mu D}{S H}$ (the inverse Peclet number, based on diffusion constant $D$ ).

In the model equations, the $\nabla \sigma$ term incorporates the effect of gradients in surfactant concentration through the constitutive relationship $\sigma(\Gamma)$ that relates surface tension $\sigma$ and surfactant concentration $\Gamma$. The choice of a particular $\operatorname{EoS} \sigma(\Gamma)$ will be a major focus of this work.

The simulations use the following values from [39], which represent typical values (the first two depend sensitively on the amount of water absorbed by the glycerol): glycerol density $\rho=1.2 \mathrm{~g} / \mathrm{cm}^{3}$, dynamic viscosity $\mu=14$ poise, gravitational acceleration $g=980 \mathrm{~cm} / \mathrm{s}^{2}$, diffusion constant $D=10^{4} \mathrm{~cm}^{2} / \mathrm{s}$, maximum (clean glycerol) surface tension $\sigma_{\max }=63.475$ dynes $/ \mathrm{cm}$, and minimum surface tension $\sigma_{\min }=37.865$ dynes $/ \mathrm{cm}$. The value of the spreading parameter $S=\sigma_{\max }-\sigma_{\min }=25.61 \mathrm{dynes} / \mathrm{cm}$ will be further discussed in Section 2.3. In order to compare simulations of inward and outward spreading, we consider for both cases a single characteristic fluid depth $H=0.7 \mathrm{~cm}$ and characteristic lateral length scale $R=3 \mathrm{~cm}$. This corresponds to typical values for fluid depth and dimension of the retaining ring in various prior experiments $[12,39,40]$. Together, these choices set the non-dimensional model parameters $\beta=2.44 \times 10^{-1}, \kappa=1.35 \times 10^{-3}$ and $\delta=7.81 \times 10^{-4}$ which we will refer to as the standard parameters.

\subsection{Initial and boundary conditions}

Simulation initial conditions are motivated by the laboratory experiments of [39] (see Figure 1). We use several variations on a standard set of basic assumptions, all radially symmetric. These choices are inspired by typical experimental parameters, but adapted here to provide a range of consistent, controlled parameter variations. The standard fluid height initial condition for both inward and outward spreading simulations is a uniform initial fluid height $h(r, 0)=1$. The standard surfactant concentration initial condition places a uniform layer of surfactant inside the retaining ring for outward spreading or outside the ring for inward spreading. In both cases, surfactant spreading occurs towards regions with less surfactant, where the surface tension is higher. 
Inward spreading initial condition IC1,

$$
h(r, 0)=1.0, \quad \Gamma(r, 0)= \begin{cases}0, & r \leqslant 1, \\ 0.7 \text { or } 2.0, & r>1,\end{cases}
$$

is used in Figures 3(a), (c), (e), (g), 4(a), (c), (e), (g), 5(a), (c), (e), (g), 6(a), (c), 7 and 9. Outward spreading initial condition IC2,

$$
h(r, 0)=1.0, \quad \Gamma(r, 0)= \begin{cases}0.7 \text { or } 2.0, & r \leqslant 1, \\ 0, & r>1,\end{cases}
$$

is used in Figures 3(b), (d), (f), (h), 4(b), (d), (f), (h), 5(b), (d), (f), (h), 6(b), 8 and 10.

In Section 3.4, we will modify these basic initial conditions to examine the effects of an annular-shaped surplus of fluid or surfactant in the vicinity of the retaining ring. This is motivated by the observation in experiments of a fluid/surfactant meniscus drawn up by the ring as the ring is slowly removed from the surface. For spreading with additional fluid thickness (Figure 11), a piecewise constant initial condition simulates the presence of additional fluid at the ring location, while the surfactant initial conditions for inward and outward spreading remain the same as defined above. We use the additional (due to meniscus) fluid height $h_{+}$as a parameter in IC3 (results shown in Figure 11):

$$
h(r, 0)= \begin{cases}1.0, & r \leqslant 1 \\ h_{+}, & 1<r \leqslant 1.5 \\ 1.0, & 1.5<r \leqslant \mathrm{~L} .\end{cases}
$$

For inward spreading with an additional surfactant in an annular region (see Figure 12), we maintain a constant initial height of the fluid, and create a region of increased surfactant concentration $\Gamma_{+}$which extends from $r=1$ out to a distance $1+r_{+}$. This is initial condition IC4:

$$
\Gamma(r, 0)= \begin{cases}0, & r \leqslant 1 \\ \Gamma_{+}, & 1<r \leqslant 1+r_{+}, \\ 0.9, & 1+r_{+}<r \leqslant \mathrm{~L} .\end{cases}
$$

Similarly, for outward spreading with an additional surfactant annulus in Figure 13, we use IC5:

$$
\Gamma(r, 0)= \begin{cases}0.9, & r \leqslant 1-r_{+}, \\ \Gamma_{+}, & 1-r_{+}<r \leqslant 1, \\ 0, & 1<r \leqslant \mathrm{~L} .\end{cases}
$$

For all simulations, we use the boundary conditions

$$
\begin{aligned}
& h_{x}=h_{x x x}=\Gamma_{x}=0, x=-L \text { and } x=L, \\
& h_{y}=h_{y y y}=\Gamma_{y}=0, y=-L \text { and } y=L,
\end{aligned}
$$

for spatial domain $[-L, L] \times[-L, L]$. There are 400 gridcells for each $2 \pi$ non-dimensional units. Grid refinement tests for this code were performed in [7]. The default value is 
$L=\pi$ and a larger domain $L=2 \pi$ is used if waves approach the boundary of the smaller domain. This domain is selected to ensure that the leading edge of the surfactant and leading fluid wave do not reach the boundary within the timescale of the simulation. This approach mimics that of the laboratory experiments, in which outward spreading data are taken before the leading surfactant edge and fluid waves approach the boundary formed by the walls of the cylindrical containment well.

Our simulation code computes with a fully 2D discretization in $(x, y)$, to enable us to monitor the results for significant deviations from axisymmetry. Surfactant droplets placed on very thin films have been observed to exhibit fingering instabilities behind the leading edge of the film $[42,43]$, but we did not observe these effects in our larger experiments. Examples of $2 \mathrm{D}$ plots are shown in Figure 3 to provide a view of the radial symmetry present in the simulations. Since we are interested in tracking the leading edge of the surfactant [7], we plot only a single independent spatial dimension $(x)$, for simplicity. The lack of fingering instabilities indicates that, in the future, 1D simulations could be performed for efficiency. For consistency with other work and because we did not know a priori that no instabilities would occur, the simulations of this paper are all computed on a $2 \mathrm{D}$ spatial domain.

\subsection{Physically motivated empirical equation of state}

As mentioned above, closing the system of equations requires an EoS relating the surface tension $\sigma$ and the surfactant concentration $\Gamma$. Previous models for the EoS have been based on the fundamental premise that surface tension decreases as surfactant concentration increases. For simplicity, the earliest versions of the model [15] employed the linear equation of state (LEoS),

$$
\sigma(\Gamma)=1-\Gamma .
$$

While this LEoS has the advantage that the constant surfactant concentration gradient simplifies the analysis of the model equations, the negative slope causes the surface tension to become negative at surfactant concentrations greater than a monolayer. This modelling choice was justified only for a range of surfactant concentrations and represents a significant and unwarranted simplification. It can be noted that in the PDE $(2.1,2.2)$, only the gradient of the EoS appears; thus, the non-negativity of the height and surfactant concentration can be imposed on the solutions.

To eliminate the restriction to submonolayer surfactant concentrations, a second model proposed in [4] uses a multilayer equation of state (MEoS), which in its dimensional form has $\tilde{\sigma}(0)=\sigma_{\max }$ and decreases asymptotically to $\tilde{\sigma}(\tilde{\Gamma})=\sigma_{\min }$ for large $\tilde{\Gamma}$. We use this $\mathrm{MEoS}$ in the non-dimensional form:

$$
\sigma(\Gamma)=(1+\eta \Gamma)^{-3}
$$

where $\eta=\sigma_{\max } / S$. Note that neither multiplicative nor additive factors affect the simulations using the MEoS, since the former are removed by non-dimensionalization, and the later by taking the gradient.

In previous work $[39,40]$, we compared simulations using the LEoS and MEoS to data from spreading experiments. This work demonstrated that neither EoS resolved 

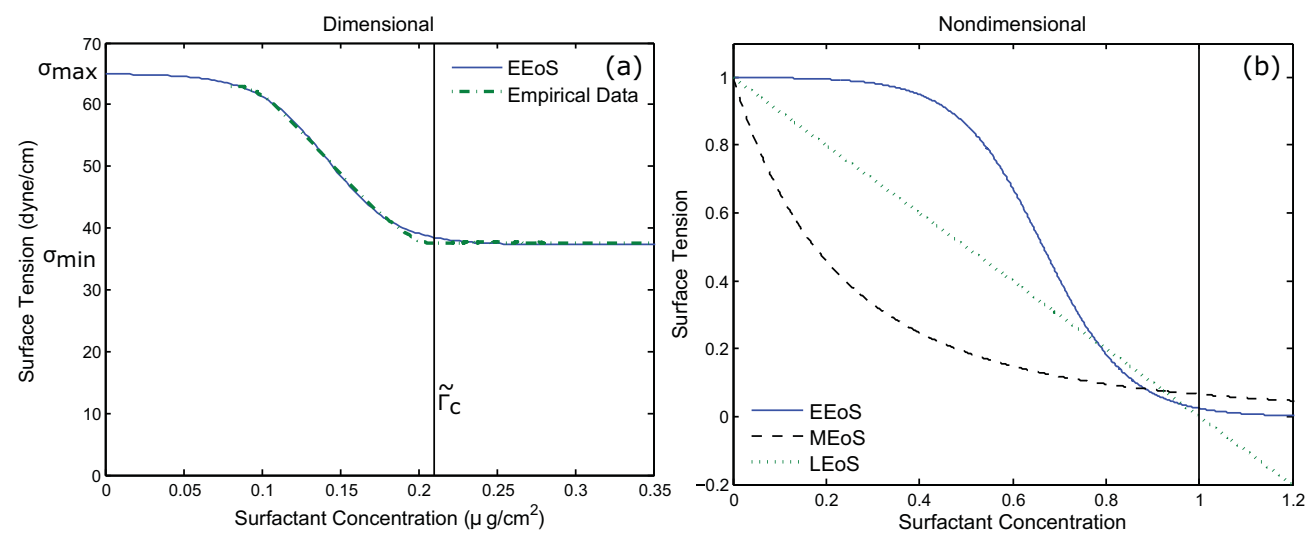

FIGURE 2. (a) Empirical data and the fit curve relating surfactant concentration and surface tension [38]. From this data, we obtain the experimental values $\sigma_{\min }=37.416$ dynes $/ \mathrm{cm}, \sigma_{\max }=$ $63.026 \mathrm{dynes} / \mathrm{cm}$ and $\tilde{\Gamma}_{c}=0.21 \mu \mathrm{g} / \mathrm{cm}^{2}$, (b) Comparison of non-dimensional linear LEoS from equation (2.5), multilayer MEoS from equation (2.6) and empirical EEoS from equation (2.7).

disagreements between simulations and experiments in either timescale or spatial distribution of surfactant. In this work, we move beyond the ad-hoc linear and multilayer choices for EoS, choose an EoS which is consistent with empirical measurements, and simulate the effect of this new choice of $\sigma(\Gamma)$ on solutions of the mathematical model for a range of experimentally realistic initial conditions. In addition, we examine the role played by the non-dimensional groups $\beta, \kappa$ and $\delta$ and test the sensitivity to initial conditions, as has long been done in studies of surfactants and thin liquid films $[9,10,14,30]$.

To obtain empirical measurements of $\tilde{\sigma}(\tilde{\Gamma})$, the standard technique is a LangmuirBlodgett trough (or Pockels scale). This apparatus measures the surface pressure while barriers compress the surfactant/lipid molecules adsorbed to the surface of a liquid. For known container dimensions and a known quantity of surface molecules, pressure and area measurements provide a plot $\tilde{\sigma}(\tilde{\Gamma})$. (Note: In the chemistry literature, the raw curve is often reported as the $\pi$ - $A$ diagram directly relating the surface pressure $\pi$ to the area occupied by the molecular monolayer.) Using this technique, it is possible draw on empirical measurements to drive the choice of a particular mathematical form of $\sigma(\Gamma)$ used in Equations (2.1) and (2.2).

To motivate a functional form for the EEoS, we examine data for a monolayer of NBD-PC on glycerol collected by Strickland [38], as plotted in Figure 2(a). A few important distinctions from the more commonly used MEoS (equation (2.6)) are worth noting. First, while the MEoS falls most steeply for low $\Gamma$ and has a single trend, the EEoS form of $\sigma(\Gamma)$ has three distinct regimes corresponding to low, intermediate and high $\Gamma$. The surface tension falls most sharply for intermediate $\Gamma$, with the low and high $\Gamma$ values remaining approximately (but not precisely) constant. Because the gradient of $\sigma(\Gamma)$ appears in Equations (2.1) and (2.2), we will see that the slopes in all three regimes have a significant impact on the simulation results.

Motivated by the experimental results, we consider a new model EoS which can capture all three regimes. While a piecewise linear function with three regimes would be analytically 
convenient (constant gradient), and capture primary features of the data except for the curvature, it has the serious disadvantage of discontinuous derivatives. In addition, the slope of the EoS should never be zero, as the model would then predict a non-physical pile-up of fluid. A negative slope would cause the same issue for large values of $\Gamma$ as the LEoS. In considering the shape of Figure 2(a), we find that a hyperbolic tangent function:

$$
\tilde{\sigma}(\tilde{\Gamma})=\frac{S}{2} \tanh \left(k_{1}\left(\tilde{\Gamma}-k_{2}\right)\right)+k_{3}
$$

models the empirical data as our EEoS while also remaining continuous and differentiable. A similar approach was used to model falling films [17,29]. We use the experimental data $\sigma_{\min }=37.416$ and $\sigma_{\max }=63.026$ from Figure 2(a) in a two-step process to obtain a modified empirical value $S=25.610$. First, an optimization routine in Matlab provides the fitted values $k_{1}=-26.31, k_{2}=0.14$ and $k_{3}=50.67$ in Equation (2.7). Second, we redefine the values of $\sigma_{\max }=k_{3}+S / 2=63.475$ and $\sigma_{\min }=k_{3}-S / 2=37.865$ so that they correspond to the fitted curve that will be used in the simulations.

We non-dimensionalize the EEoS using $\Gamma=\tilde{\Gamma} / \tilde{\Gamma}_{c}$ and $\sigma=\left(\tilde{\sigma}-\sigma_{\min }\right) / S$. The critical surfactant concentration $\tilde{\Gamma}_{c}$ is the value of $\tilde{\Gamma}$ at which the trough data indicate that additional surfactant does not further reduce the surface tension. This value is obtained by finding the local minimum in the data at $\tilde{\Gamma}_{c}=0.21$ in the EEoS (see vertical lines in Figure 2). This is a lower value than previous papers, which used 0.3 as an approximation of $\tilde{\Gamma}_{c}$. To provide a consistent comparison, we will use an MEoS (see equation (2.6)) derived from the same values for $\sigma_{\max }, \sigma_{\min }, \tilde{\Gamma}_{c}$ and $S$, with $\eta \equiv \sigma_{\min } /\left(\sigma_{\max }-\sigma_{\min }\right)=1.48$. Figure 2(b) has all three curves, the LEoS, MEoS and EEoS.

We have explored simulations using the value of $S$ as the slope of the EEoS at the inflection point instead of the maximum difference in surface tensions in the hope that the larger value (nearly double) would improve the agreement in timescale between simulation and experiment. Since this was not the case, we have not included the simulations here.

The simulations of this paper are performed using an open-source code described in [18], with code and documentation freely available on Github [19]. In previous work by this group and others, model equations (2.1), (2.2) have been solved using many approaches $[39,44,45]$; the advantage of our code is that it facilitates easy modification of terms in the equation and boundary conditions and provides a package for convergence testing. The second-order scheme is based on a finite volume approach (using Newton's Method and BiCGStab), which takes advantage of the free open source Clawpack package (www.clawpack.org) and enables the user to compute solutions with small (or zero) coefficients on the regularizing terms.

\section{Results}

We present numerical solutions to the system of PDE (2.1,2.2) using an empirically derived EoS, realistic initial and boundary conditions, and appropriate model parameters $\beta, \kappa, \delta$. We frame our investigations as answers to four key questions:

(1) Section 3.1 Choice of EoS shape: How do the general dynamics of solutions for the $\mathrm{MEoS}$ differ from those for the EEoS? 
(2) Section 3.2 Offsets to the EoS: What is the effect of translating the EEoS horizontally (offset in $\Gamma$ )? (Translating the EEoS vertically (offset in $\sigma$ ) is known to have no effect, since only gradients in $\sigma$ matter.)

(3) Section 3.3 Dependence on non-dimensional parameters: What is the effect on simulations with the EEoS of varying the non-dimensional parameters?

(4) Section 3.4 Effect of the retaining ring: Do simulations with the EEoS indicate that the retaining ring that creates the initial surfactant distribution has a strong effect on the spreading dynamics?

We answer these questions by focussing on two features also observable in experiments: The fluid height $h_{c}(t) \equiv h(0, t)$ at the centre of the domain (which is affected by the capillary ridge) and the location $r_{s}(t)$ of the leading edge of the surfactant as it spreads. In addition, we discuss the implications of these results for future laboratory experiments.

In viewing simulation results, keep in mind that they are computed in two spatial dimensions $(x, y)$, but plotted as $h(x, t)$ and $\Gamma(x, t)$. As noted above, for symmetric initial conditions such as ours, deviations from axisymmetry are not significant [7]. Also note that because the size of surfactant molecules is insignificant compared to that of the fluid depth, the model assumes the surfactant does not add to the height in the fluid/surfactant system. Thus, the $\Gamma(x, t)$ plots represent the local surface concentration across the diameter of the well; physically, this corresponds to a more-densely or less-densely packed surfactant layer. That is, a larger $\Gamma(x, t)$ value means that locally there are more surfactant molecules at that point in time.

All simulations are run with the standard parameters defined at the end of Section 2.1 unless otherwise noted. The solutions to the surfactant equation do not have compact support due to diffusion ( $\delta>0$ in the model, as well as in reality). Therefore, we must choose an effective location of the leading surfactant front, which we define as the location where $\Gamma=0.01$.

\subsection{Choice of EoS shape}

We begin by answering the most general question: What are the most significant effects of choosing the EEoS in place of the more commonly used MEoS? Figures 3 and 4 show typical results at above and below the critical monolayer concentration, respectively. In both figures, we present solutions for inward (a), (c), (e), (g) and outward (b), (d), (f), (h) surfactant spreading. The top quartet of plots (a)-(d) presents simulations using the MEoS and the bottom quartet (e)-(h) presents simulations using the new EEoS. Each profile is a snapshot in time.

\subsubsection{Inward spreading}

During inward spreading, the fluid develops an inward-moving annular capillary ridge as it is pulled by the surfactant spreading into the central (clean) region (Figures 3(a), (c), (e), (g) and 4(a), (c), (e), (g)). The fluid ridge coalesces into a single central maximum and then relaxes to an equilibrium at the original uniform height $(h(x, t)=1)$. These general dynamics of fluid coalescence, central growth and decay were previously observed 

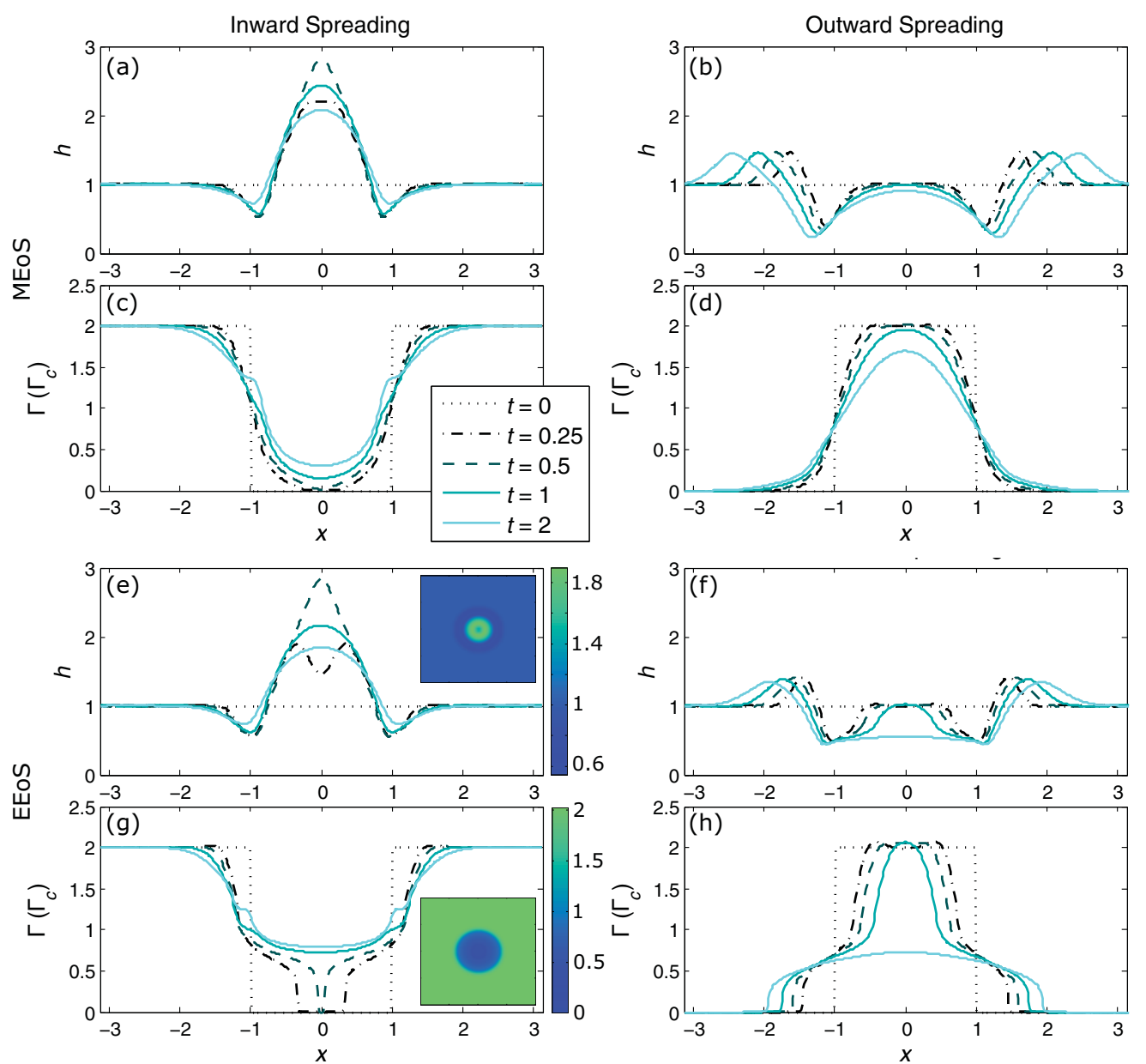

FiguRE 3. Typical spreading dynamics for each equation of state with standard parameters and initial surfactant concentration $\Gamma=2.0>\Gamma_{c}$. The upper quartet of plots (a)-(d) use the MEoS whereas the lower quartet (e)-(h) use the EEoS. Plots (a), (b), (e), (f) are fluid profile dynamics $h(x, t)$ and $(\mathrm{c}),(\mathrm{d}),(\mathrm{g}),(\mathrm{h})$ are surfactant concentration profiles $\Gamma(x, t)$. Left plots (a), (c), (e), (g) have an inward spreading initial condition (IC1) and right plots (b), (d), (f), (h) have an outward spreading initial condition (IC2). Inserts at the right-hand side of plots (e) and (g) show the full 2D EEoS simulation with IC1 at time $t=0.25$. For a comparison to outward spreading experiments above $\tilde{\Gamma}_{c}$, see [12] Figure 2 and [40] Figure 9.

for submonolayer concentrations in laboratory experiments using laser profilometry [12, $39,40]$, and are similar for either choice of EoS. This result explains why in [39] there was surprisingly good morphological agreement in the experiment and simulation fluid profiles with the MEoS despite the poor agreement in timescale and surfactant distribution. However, there is an important distinction in $h(x, t)$ : For simulations run with the MEoS (Figures 3(a), (e) and 4(a), (e)), the annular fluid capillary ridge (double-peaked structure) coalesces more quickly than those with the EEoS. 


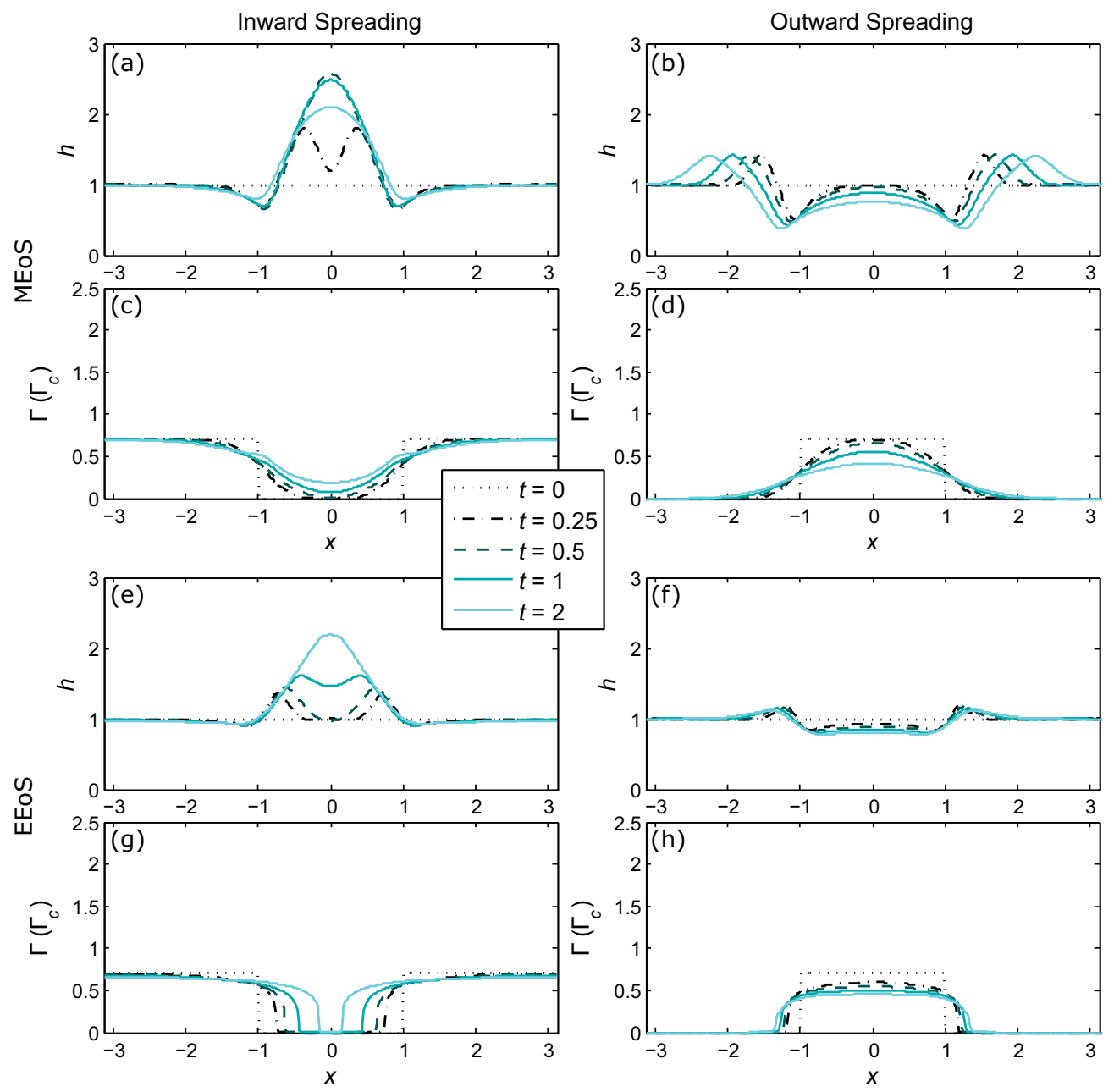

FIGURE 4. Typical spreading dynamics for each equation of state with standard parameters and initial surfactant concentration $\Gamma=0.7<\Gamma_{c}$. The upper quartet of plots (a)-(d) use the MEoS whereas the lower quartet (e)-(h) use the EEoS. Plots (a), (b), (e), (f) are fluid profile dynamics $h(x, t)$ and plots (c), (d), (g), (h) are surfactant concentration profiles $\Gamma(x, t)$. Left plots (a), (c), (e), (g) have an inward spreading initial condition (IC1) and right plots (b), (d), (f), (h) have an outward spreading initial condition (IC2). For a comparison to experiments below $\tilde{\Gamma}_{c}$, see [39] Figure 7 (inward spreading, distension growth), Figure 11 (inward spreading, distension decay) and [40] Figure 10 (outward spreading stalls).

The surfactant concentration profiles $\Gamma(x, t)$ are more distinct for the two EoS choices. For surfactant layers with an initial condition above $\Gamma_{c}$ (Figure 3), the EEoS surfactant profile has a leading "foot" that pushes into the central region, with a pronounced and steep leading edge. In contrast, the MEoS surfactant curve retains a smoother profile at all times, with only a small kink near the location of the ring at radius $r=1$ (associated with the dip in the fluid at that location). Note that this is different than the precursor "foot" observed in [41], which was a result of interaction between surfactant and a solid 
substrate, not surfactant and thin liquid film. Even for surfactant layers with an initial condition below $\Gamma_{c}$ (Figure 4), the EEoS is better able to maintain strong gradients than the MEoS. Experiments for inward spreading have not been performed with $\tilde{\Gamma}>\tilde{\Gamma}_{c}$; this is a prediction that could be tested in future experiments. In the figures that follow, we will focus on the parameters used in Figure 3 (initial conditions with more than a monolayer of surfactant) since the surface flow in this case is more sensitive to the choice of EoS due to the simultaneous presence of regions of high, middle and low surfactant concentrations.

\subsubsection{Outward spreading}

During outward spreading, the fluid develops an outward-moving annular capillary ridge as it is pushed by the surfactant spreading into the outer (clean) region (Figures 3(b), (d), (f), (g) and 4(b), (d), (f), (g)). This feature is present independent of the choice of EoS, and for initial surfactant concentrations above and below $\Gamma_{c}$. However, for the MEoS simulations, the height $h_{c}$ of the central peak decays slowly even for simulations run much longer, whereas with the EEoS, there is a central fluid depression that extends across the entire region in which the surfactant had initially been deposited. The EEoS behaviour is consistent with what is observed in experiments [40], and thus is a better model.

As with inward spreading, the surfactant concentration profiles $\Gamma(x, t)$ are even more distinct for the two EoS choices. Again the surfactant layers with an initial condition above $\Gamma_{c}$ produce a foot-like layer that emerges from the central region, ending in a pronounced leading edge. The sharp decrease in surfactant concentration coincides with the location of the fluid capillary ridge. Below $\Gamma_{c}$ the foot layer extends with no reservoir. As shown in Figures 1(b), (c), these same morphologies are present in the experiments. In the experimental (top view) images above $\tilde{\Gamma}_{c}$, a bright central region is surrounded by a lower intensity foot behind the leading edge. In the images below $\tilde{\Gamma}_{c}$ no reservoir is present. These important features are not reproduced by the MEoS-based simulations. Instead, the MEoS case shows a consistent shape as the initial central surfactant layer decays and the leading edge seems to show little evolution. Therefore, the reservoir and foot-like features provide a striking improvement in morphological agreement between experiment and simulation by using the EEoS as compared to the MEoS.

\subsubsection{Timescale}

In all cases (inward/outward, MEoS/EEoS), the fluid profile maintains its initial depression at the surfactant boundary in the initial condition (corresponding to the retaining ring location in the experiment). In Figures 3(b), (f), the outward spreading central height evolution is much more distinctive between choices of EoS than in the inward spreading simulations. The MEoS has a smooth decay over time, while the EEoS has a growth phase and a steep decay phase. The surfactant leading edge plots also are more distinct; they have similar shapes, but the leading edge with the EEoS has a lower velocity.

Figure 5 illustrates how the key dynamics from Figures 3 and 4 can be captured by considering only the height of the central peak $\left(h_{c}\right)$ and the location of the leading 

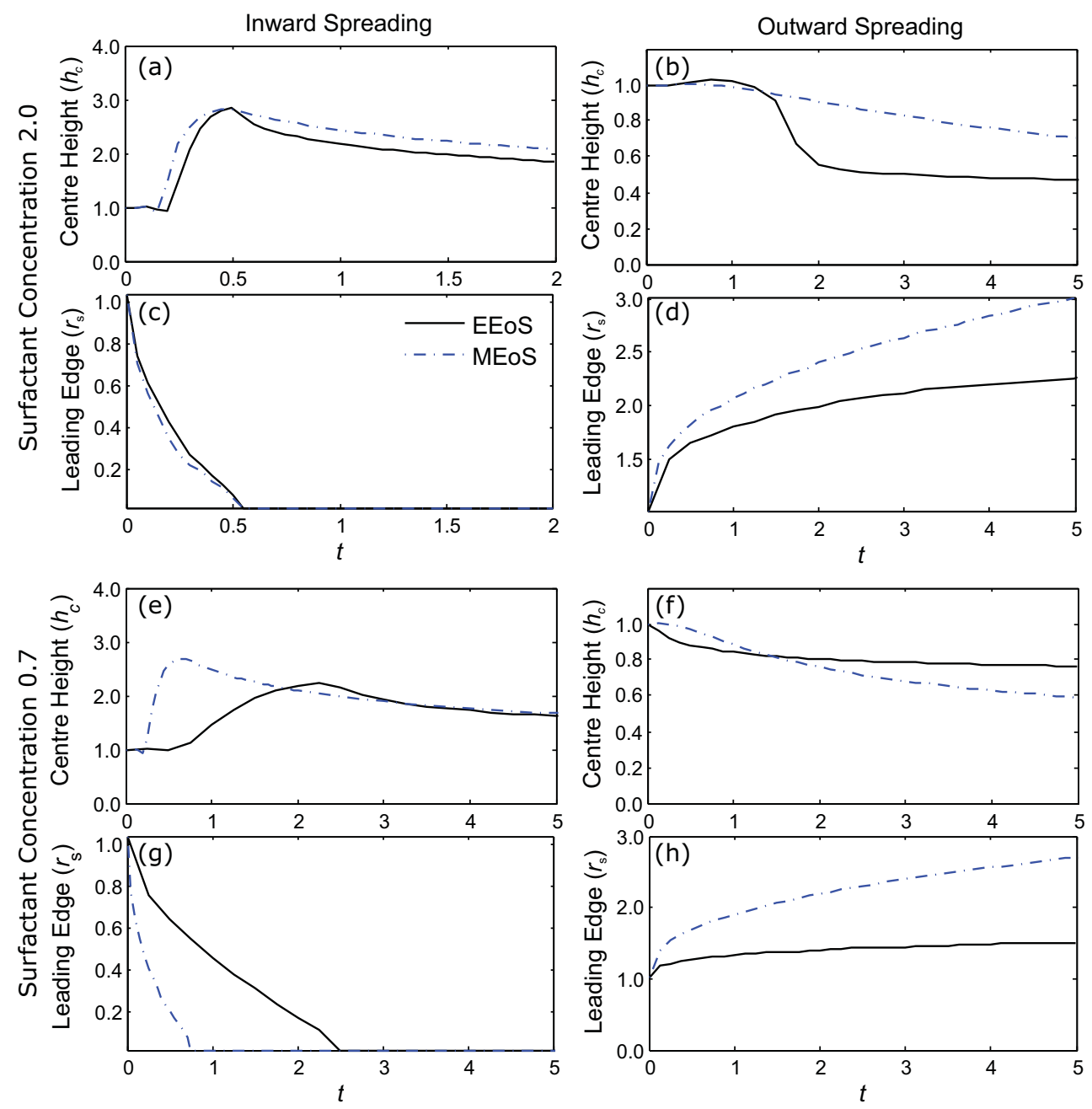

Figure 5. Comparison of $h_{c}(t)(\mathrm{a}),(\mathrm{b}),(\mathrm{e}),(\mathrm{f})$ and $r_{s}(t)(\mathrm{c}),(\mathrm{d}),(\mathrm{g})$, (h) for simulations of Figures 3 and 4. For a comparison to experiments, see [39] Figures 6, 9 and 12 (inward spreading) and [12] Figures 3 and 4, and [40] Figures 8 and 12.

edge of surfactant $\left(r_{s}\right)$. These plots compare the effect of MEoS and EEoS on these two dynamics. Importantly, they do not agree with each other. This suggests the need for a second timescale beyond $\tilde{t}=\left(\frac{\mu L^{2}}{S H}\right) t$, the one used in the non-dimensionalization. This situation arises because only the gradient of the EoS appears in the system of PDE (2.1), (2.2). In the LEoS, this gradient is negative and constant, in the MEoS the gradient is negative and gradually decreasing, and in the EEoS there are three distinct regimes. In the EEoS, at low and high $\Gamma$, the gradient is negative and small, but for intermediate surfactant concentrations, the gradient changes dramatically in magnitude. It is thus unlikely that a single parameter $S$ captures the magnitude of the gradient, and 
therefore there is no single timescale. This observation may explain why, in all previous comparisons of simulations and experiments, it has been necessary to redimensionalize the simulations using a different (shorter by a factor of 2 to 10) timescale than the model would predict $[38,40]$.

To further investigate the timescale, in Figure 6 we plot the spreading of the leading edge of surfactant $r_{s}$ as a function of $t-t_{c}$ where $t_{c}$ is closure time. We see power law relationships with spreading rates similar to the predictions in [20]: An exponent of 0.25 for outward spreading (Figure 6(c)) and 0.81 for inward spreading (Figure 6(b)). In outward spreading experiments [40], we similarly observed an exponent of 0.25 ; for inward spreading exponents [39], the exponent was 0.81 , with a similar, steeper, trend at short times. Note that in the inward spreading plot, later times are on the left side of the plot; this is the regime in which we expect agreement. Also, as suggested in [20], in both the inward and outward spreading plots the spreading exponent is controlled more by the type of EoS (MEoS versus EEoS) than the initial surfactant concentration. In the outward spreading case, the spreading exponent is larger for the MEoS than the EEoS, which we would expect since for low surfactant concentrations, the MEoS has a larger gradient than the EEoS.

\subsection{Offsets to the EoS}

The choice of a particular lipid will determine a unique EoS, specific to that lipid [23,35]. However, the general shape shown in Figure 2 exhibits many features common to a number of lipids. Therefore, it is important to ask what features of the solutions change when performing a mathematical transformation that translates the EEoS horizontally, which changes, for example, the value of $\Gamma$ at which the surface tension is maximal. To exemplify a few basic behaviours, we perform simulations in which we mathematically translate the EEoS $30 \%$ each direction: to the left $\sigma(\Gamma+0.3)$, right $\sigma(\Gamma-0.3)$, up $\sigma(\Gamma)+0.3$ and down $\sigma(\Gamma)-0.3$. Because only the gradient of the EoS appears in the model system of partial differential equations (see Equations (2.1), (2.2)), vertical translations do not affect the results. Understanding the effects of these translations allow us to test which features of the chosen EoS are essential for making quantitative comparisons with experiments.

Inward spreading: As shown in Figure 7(a), the simulations with the $\operatorname{EEoS} \sigma(\Gamma+0.3)$ have a smaller dip at the centre as compared with the EEoS translated to the right. In addition, the EEoS translated to the left (a) has an earlier coalescence of the annular capillary fluid ridge compared to (b). In the surfactant profiles $\Gamma(x, t)$ (panels $\mathrm{c}, \mathrm{d}$ ), the left-translated EEoS creates a smaller concentration $(\approx 0.25)$ in the foot, whereas for the right-translated EEoS, the foot concentration is about 0.7. As expected, there is no difference for vertical translations and small differences for horizontal translations (see panels e, f).

Outward Spreading: We observe that outward spreading is much more sensitive to horizontal offsets to the EoS than inward spreading, as shown in Figure 8. Here, we additionally include larger $(60 \%)$ translations in the location of $\Gamma_{c}$, and do not consider vertical translations because they have no effect. We observe that both $\operatorname{EEoS} \sigma(\Gamma+0.3)$ and $\sigma(\Gamma+0.6)$ lead to solutions that look much like the central height and leading edge 

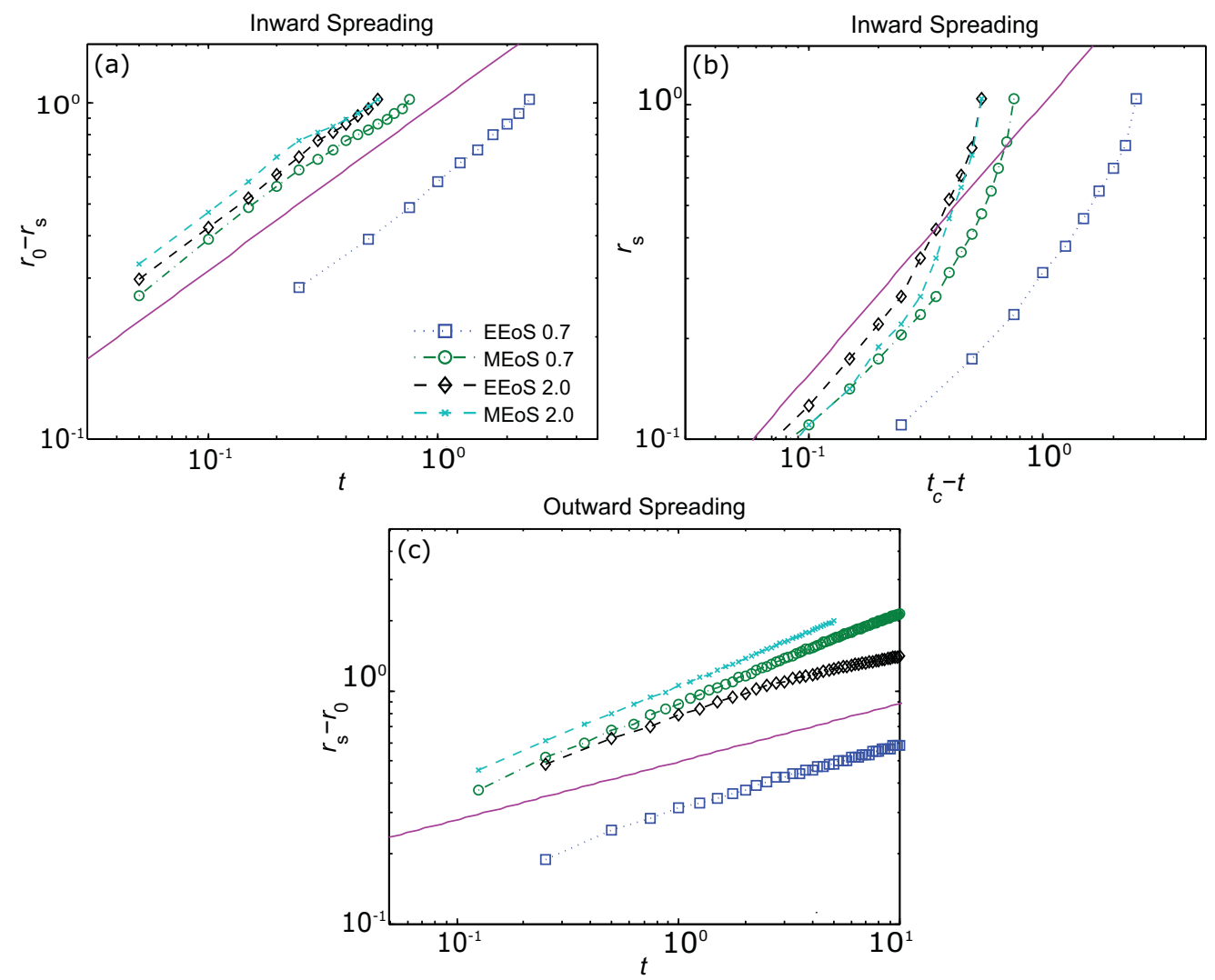

FiguRE 6. (a) The inward spreading long-time dynamics of $r_{0}-r_{s}$ as a function of $t$, using data from Figures 5(c), (g). The solid magenta line $\left(r_{0}-r_{s}\right) \propto t^{0.5}$ is a visual reference, (b) The inward spreading long-time dynamics of $r_{s}$ as a function of $t-t_{c}$, using the same data from Figures 5(c), (g) as in (a), where $t_{c}$ is the closure time (and late times are at the left side). In this visualization, the solid magenta line $\left(r_{0}-r_{s}\right) \propto t^{0.81}$ provides a comparison to similarity solutions in [20], (c) The outward spreading long-time dynamics of $r_{s}-r_{0}$ as a function of $t$, using data from Figures 5(d), (h). The solid magenta line $\left(r_{0}-r_{s}\right) \propto t^{0.25}$ provides a comparison to similarity solutions in [20].

results for the MEoS of Figure 5. In both cases, the central height $h_{c}(t)$ has a consistently small gradient and the surfactant leading edge $r_{s}(\mathrm{t})$ advances more rapidly than for the original $\sigma(\Gamma)$. Note that this similarity occurs because left translations move the region of the function with steeper gradient to low surfactant concentrations (such as those near $\left.r_{s}(\mathrm{t})\right)$, which makes its gradient more like that of the MEoS.

Translating the function to the right, $\sigma(\Gamma-0.3)$ and $\sigma(\Gamma-0.6)$, moves the steeper portion of the EoS to larger surfactant concentrations. For our initial conditions, this accelerates the fluid growth and decay phases so that $h_{c}(t)$ is quite steep, especially in the decay phase. However, the leading edge of the surfactant $r_{s}(t)$ (where the surfactant concentration $\Gamma$ is lower) advances more slowly, since the large gradient in the EoS has been moved to larger surfactant concentrations. 

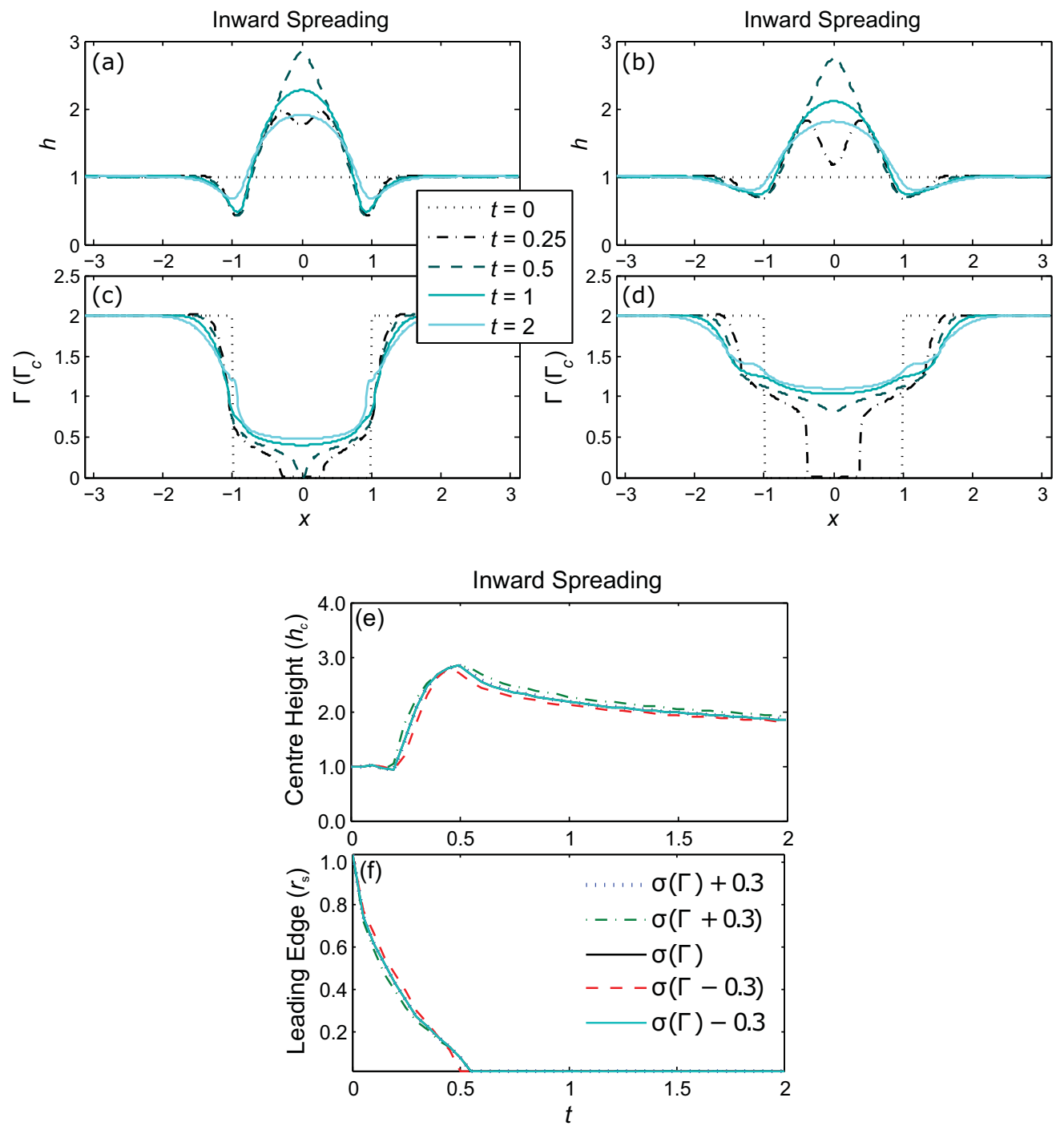

FIGURE 7. Dynamics for standard parameters and initial condition (IC1) using EEoS $\sigma(\Gamma+0.3)$ in plots (a), (c) and EEoS $\sigma(\Gamma-0.3)$ in plots (b), (d). Characterizing (e) $h_{c}(t)$ and (f) $r_{s}(t)$ for representative translations of the EEoS.

\subsection{Dependence on non-dimensional parameters}

The values for the non-dimensional parameters $(\beta, \kappa, \delta)$ in the model are derived from fluid and physical properties of the system, and in general cannot be independently varied in experiments. Simulations provide a means to test the effects of each. We performed simulations for one-quarter, half and double the standard parameter values for $\beta, \delta$ and $\kappa$, and found that the only notable changes occurred as a function of $\beta$, which we will explore here for both inward and outward spreading. These effects would be difficult to detect in experiments. Because $\beta \equiv \frac{\rho g H^{2}}{S}$, the only way to change $\beta$ without changing the 

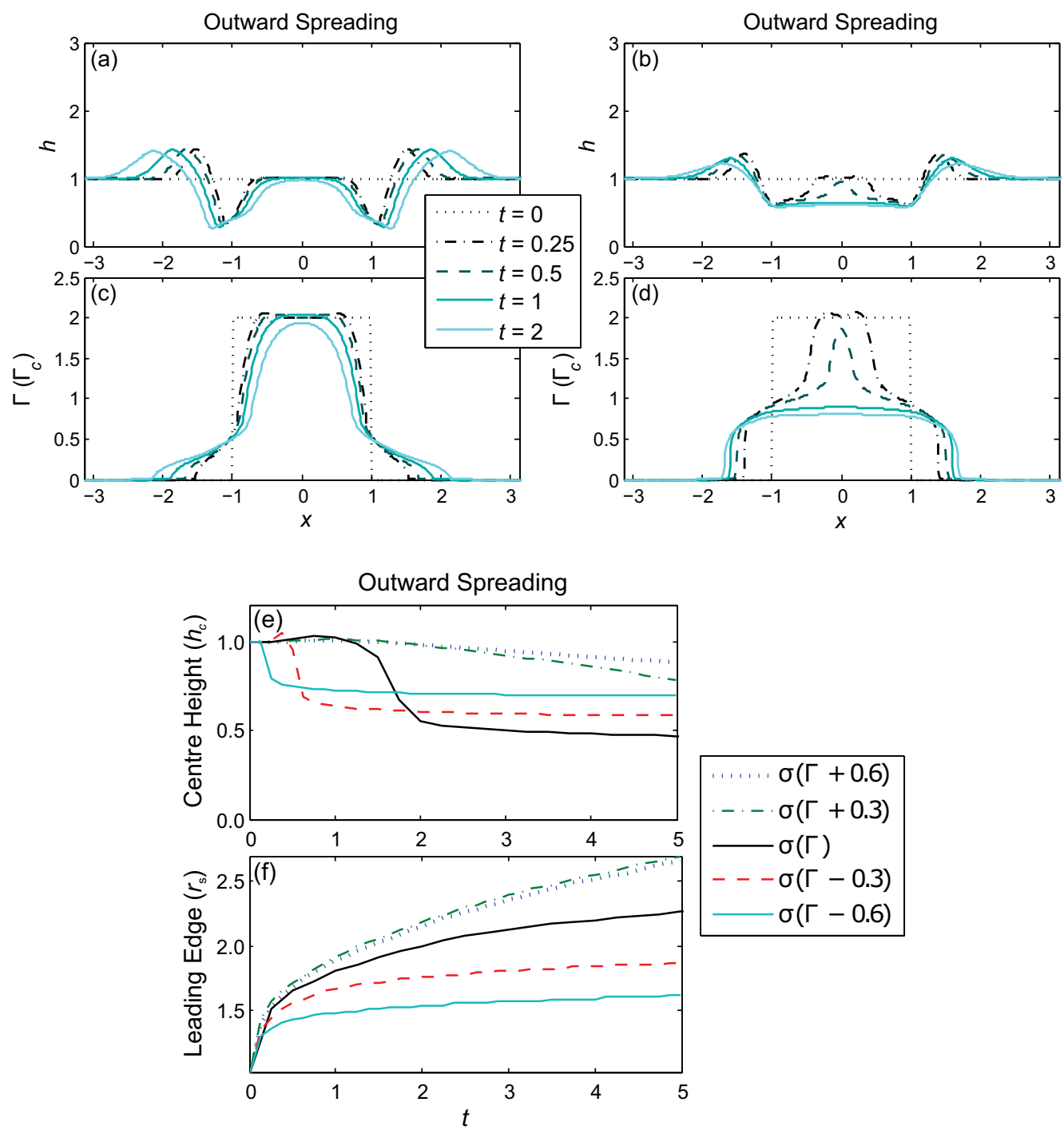

FIGURE 8. Outward spreading sample dynamics for standard parameters and initial condition (IC2) using $\operatorname{EEoS} \sigma(\Gamma+0.3)$ in plots (a), (c) and $\operatorname{EEoS} \sigma(\Gamma-0.3)$ in plots (b), (d). (e), (f) Characterizing (e) $h_{c}$ and (f) $r_{s}$ for the EEoS $\sigma(\Gamma \pm 0.3)$ and $\sigma(\Gamma \pm 0.6)$ with standard parameters.

other parameters is to choose a fluid of a different density $\rho$, but this choice of a new material would also change $S$.

Inward spreading: As shown in Figures 9(a), (b), the dynamics of $h_{c}$ and $r_{s}$ are qualitatively similar, independent of $\beta$. There are some small differences for a particular choice of $\beta$ : Smaller values (lower gravity, density or $H$ compared to spreading parameter $S$ ) can produce a larger fluid peak at the centre, more rapidly. We can quantify the similarity by considering the relaxation from a central peak at time $t_{\text {peak }}$ back to a uniform fluid height 

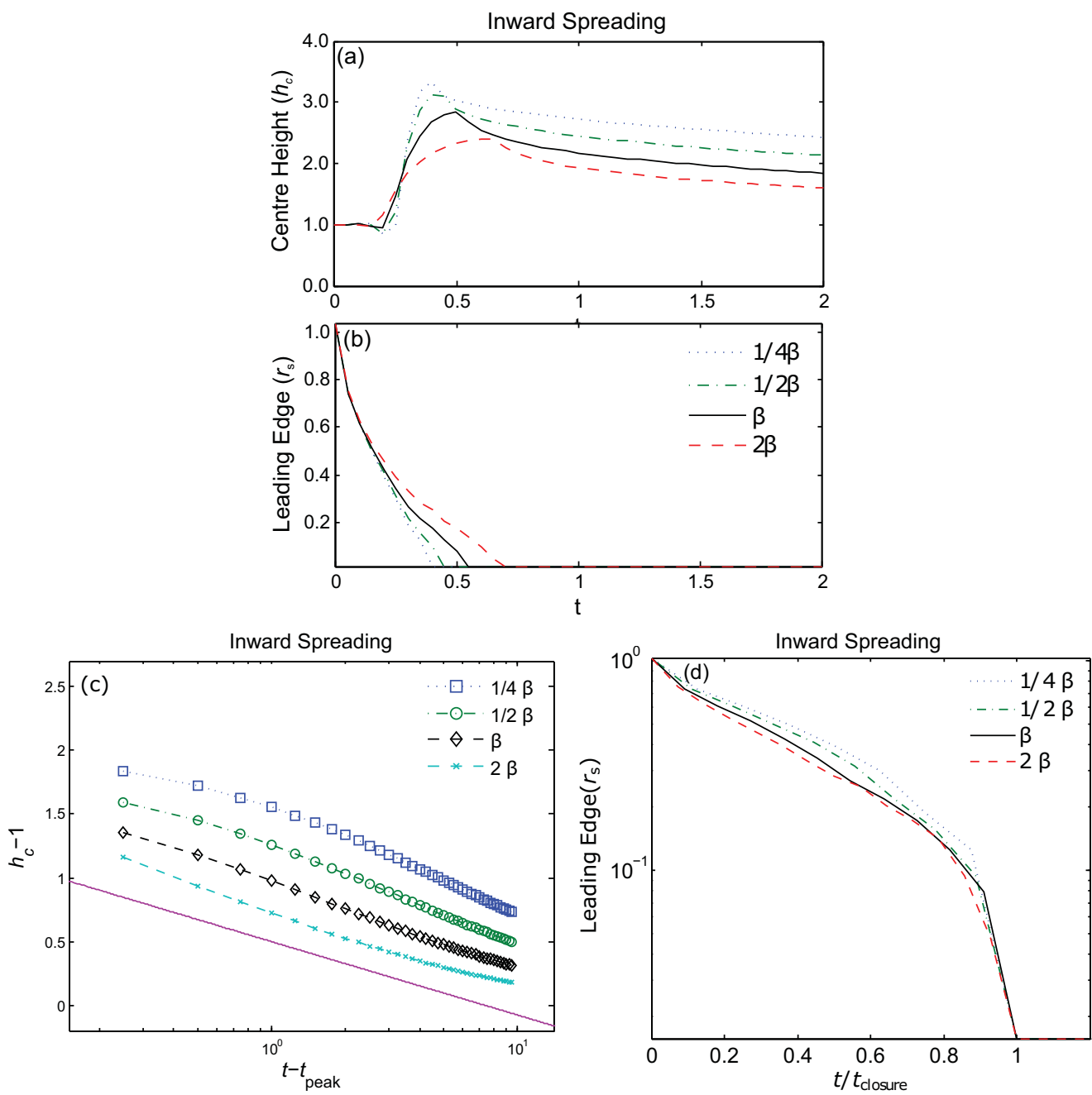

FIGURE 9. Inward spreading simulations at fixed initial condition (IC2), varying the relative importance of gravitational and capillary forces, through the non-dimensional parameter $\beta$. Plots of (a) central fluid height $h_{c}(t)$ and (b) surfactant leading edge location $r_{s}(t)$. Self-similar behaviour of the decay of the fluid at centre (c) and motion of surfactant leading edge (d). The time $t_{\text {peak }}$ is defined as the time when $h_{c}(t)$ is at its maximum value and $t_{\text {closure }}$ is defined as the time when the leading edge location $r_{s}=0$. The solid magenta line in (c) given by $e^{h_{c}-1} \propto\left(t-t_{\text {peak }}\right)^{-0.25}$ is a visual reference and can be compared to similarity solutions in [20].

$h(x, t)=1$. We observe that these dynamics follow a logarithmic decay (see Figure 9(c)). Finally, we rescale the dynamics in Figure 9(b) by taking the time for the surfactant to reach $r_{s}=0$ to be a characteristic closure time. As shown in $9(\mathrm{~d})$, under this rescaling, the $r_{s}(t)$ dynamics are also independent of the choice of $\beta$.

Outward spreading: Similar effects are observed for outward spreading in Figure 10, with smaller $\beta$ resulting in a taller capillary ridge, but with a largely invariant timescale 


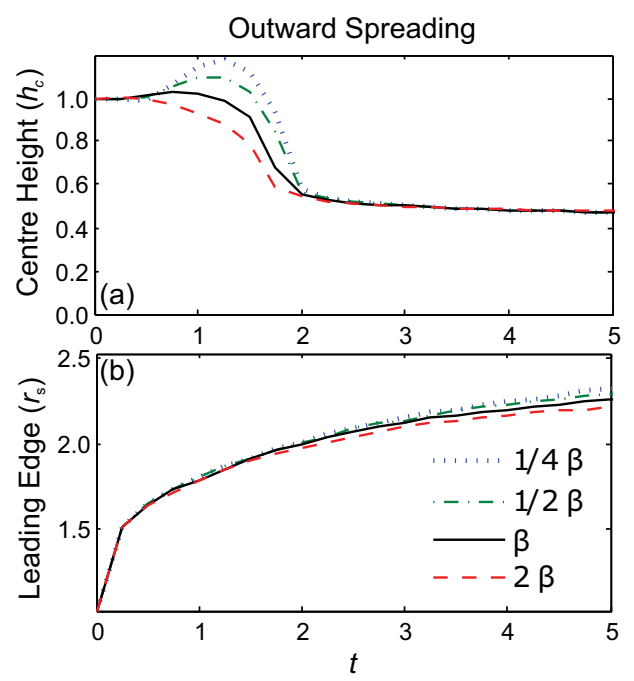

FIGURE 10. Outward spreading simulations with nonstandard parameters to show the effect of varying $\beta$ on outward spreading using initial condition (IC3). Plots of (a) central fluid height $h_{c}(t)$ and (b) surfactant leading edge location $r_{s}(t)$.

(compare Figure 10 to Figure 9 where the timescale varies). A slight trend of faster spreading for lower $\beta$ is also present.

\subsection{Effect of retaining ring}

The first three investigations focus on solutions of the mathematical model, which has been compared to experiments. This final investigation uses simulations to investigate the influence of the experimental apparatus. A key difference between experimental and numerical investigations is the necessity of using a retaining ring to set up the initial conditions when performing experiments. When this ring is lifted, a meniscus forms and then releases back to the surface after pinch-off. This raises the possibility that additional fluid or surfactant is present at the original location of the retaining ring, and that this excess could influence the dynamics. Below, we perform numerical tests which establish that this is unlikely to be the case. Initial conditions (IC3-5) are chosen to mimic conditions that could occur experimentally, and we test whether these conditions could have a significant impact on the timescale of the dynamics, as well as the spatial distribution of the surfactant. The negative results of these studies give credence to the idea [40] that the excess light intensity just-visible at the leading edge of the outward spreading images in Figures 1(b), (c) is instead due to fluorescence resonance energy transfer [37] rather than excess surfactant.

\subsubsection{Fluid annulus}

We first consider the case of starting from an initial condition which places an additional annulus of fluid at the location of the ring, to mimic the after-effects of meniscus pinch-off. As shown in Figure 11, even adding $10 \%$ or $20 \%$ of additional fluid results in only a minor 

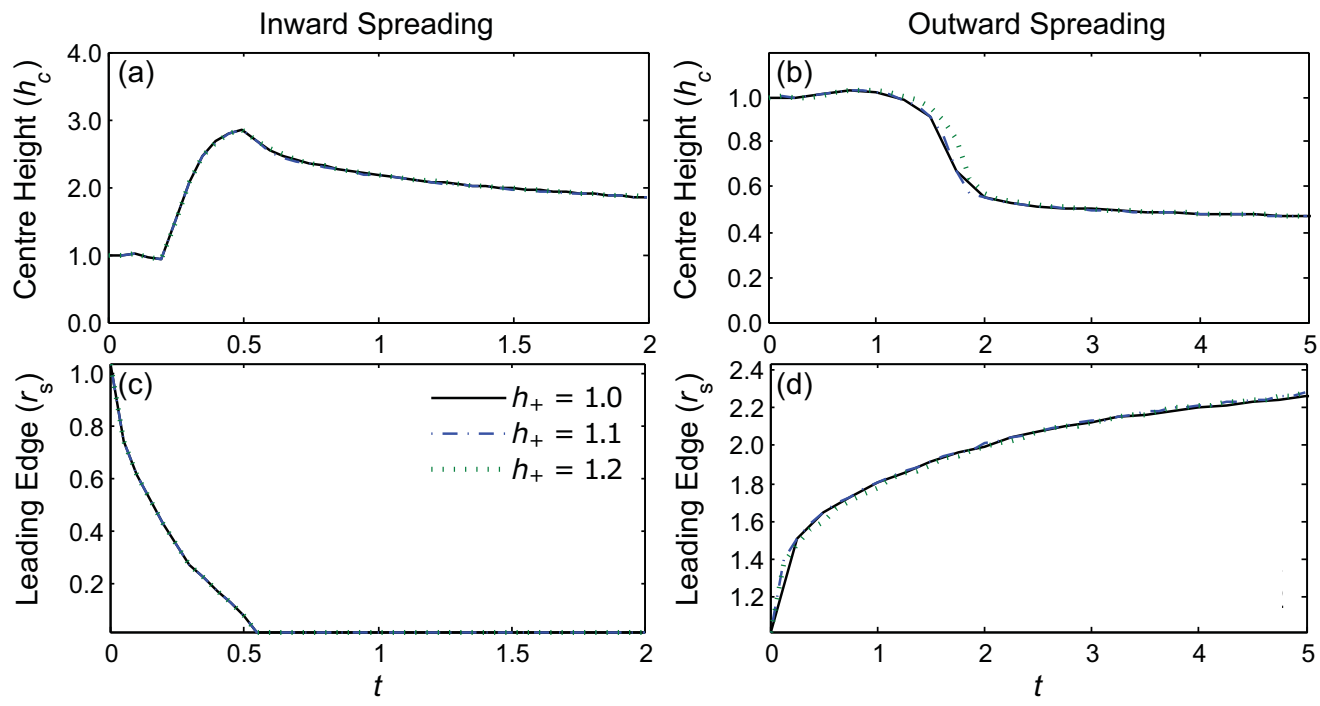

FiguRE 11. Inward and outward spreading simulations with standard parameters and initial condition (IC3) comparing central fluid height $h_{c}(t)$ (a), (b) and surfactant leading edge $r_{s}(t)$ (c), (d) for no fluid annulus and with an annulus $10 \%$ and $20 \%$ above the original level.

change to the $h_{c}$ and $r_{s}$ dynamics, independent of whether inward or outward spreading is considered. Thus, although the ring visibly lifts a meniscus of fluid in the experiments, these simulations suggest that this effect is unlikely to affect spreading dynamics for inward or outward spreading.

\subsubsection{Surfactant annulus}

Because the ring pulls up an annular meniscus of fluid over the span of many minutes, surfactant has time to accumulate at this interface. When the meniscus pinches off, it could therefore leave behind an annular region with a surplus of surfactant. An annulus of surfactant will have the peculiar effect of superimposing both inward and outward spreading at the inner and outer edges of the annulus, respectively. This effect is in addition to the underlying surfactant gradient due to the original inward or outward initial conditions. As will be shown below, this will impact the spreading dynamics.

Inward Spreading: Figure 12 shows the results of inward spreading simulations obtained by varying both the concentration and width of the annular region. Increasing the concentration of a fixed annulus width $r_{+}=0.25$ or width of a fixed annulus concentration $\Gamma_{+}=2.0$ increases the velocity of both growth and decay dynamics. The larger annulus produces a larger central fluid maximum height $h_{c}$ at an earlier time in Figures 12(c), (d) and more rapid inward surfactant spreading in Figures 12(e), (f). With a large annulus concentration, excess surfactant acts as a reservoir (the EEoS has a very small gradient at large surfactant concentrations).

Outward spreading: Figure 13 illustrates the effect of an annulus of surplus surfactant on the outward spreading dynamics. As in the inward spreading case, larger surfactant 

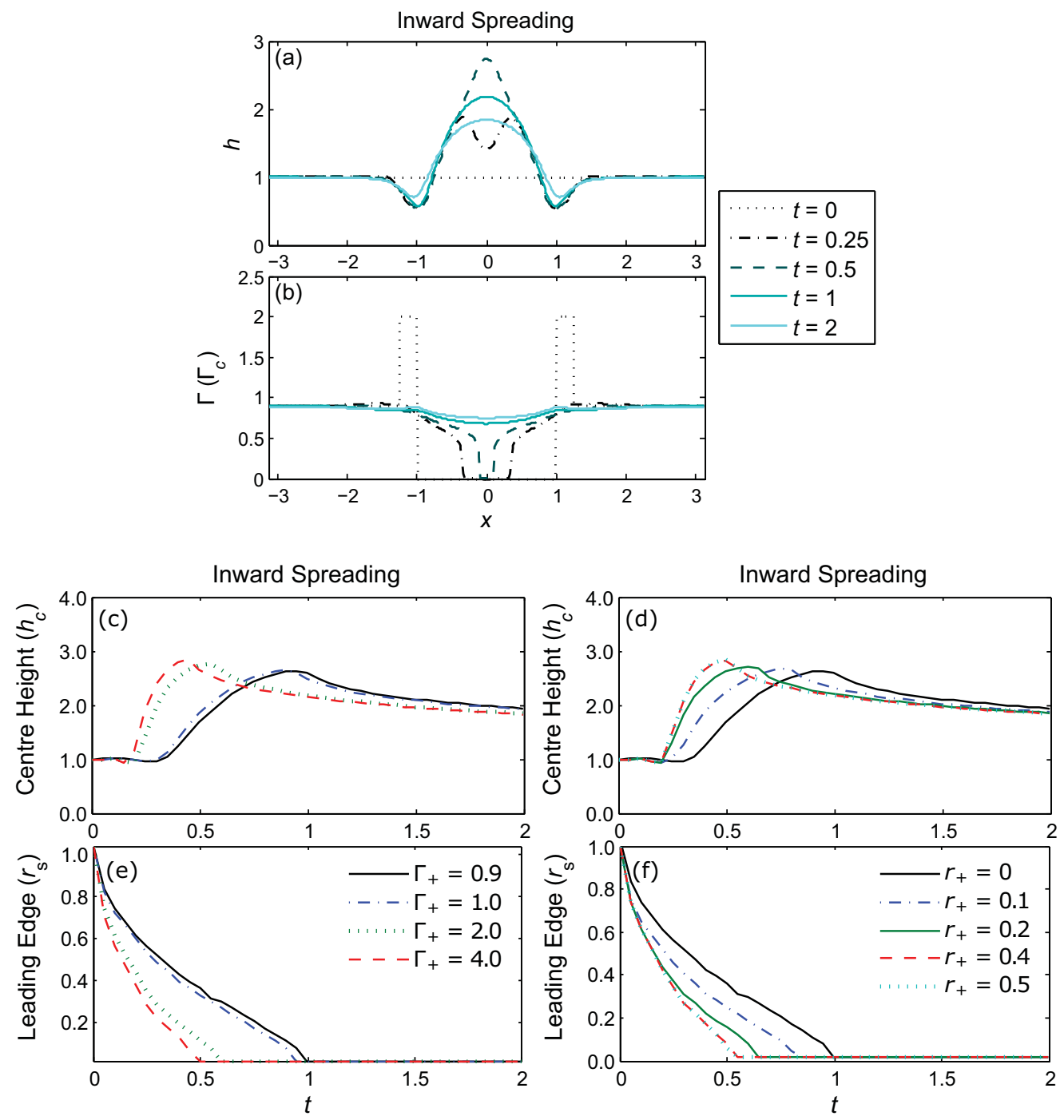

FIGURE 12. Inward spreading with additional annulus of surfactant at the ring location. (a), (b) Standard parameters and initial condition (IC4) using a larger initial surfactant concentration $\left(\Gamma_{+}=2.0\right)$ extending $r_{+}=0.25$ beyond the ring location. The effect of additional surfactant on $h_{c}$ and $r_{s}$ for (c), (e) the same parameters, but with varying $\Gamma_{+}$and (d), (f) the same parameters but varying $r_{+}$.

volumes (whether via increased annulus concentration in Figures 13(c), (e) or increased width in Figures 13(d), (f)) produce a longer relaxation time for the fluid in the centre, and more rapid outward spreading of surfactant. Note that for a large annulus concentration $\left(\Gamma_{+}=4.0\right)$, the additional inward spreading is most obvious. The central fluid height increases as long as the annulus of surfactant is still present, decreasing only once the surplus has spread outward. Smaller volumes of surfactant equilibrate almost immediately, thus those curves are essentially monotonic while those for larger volumes are not. 

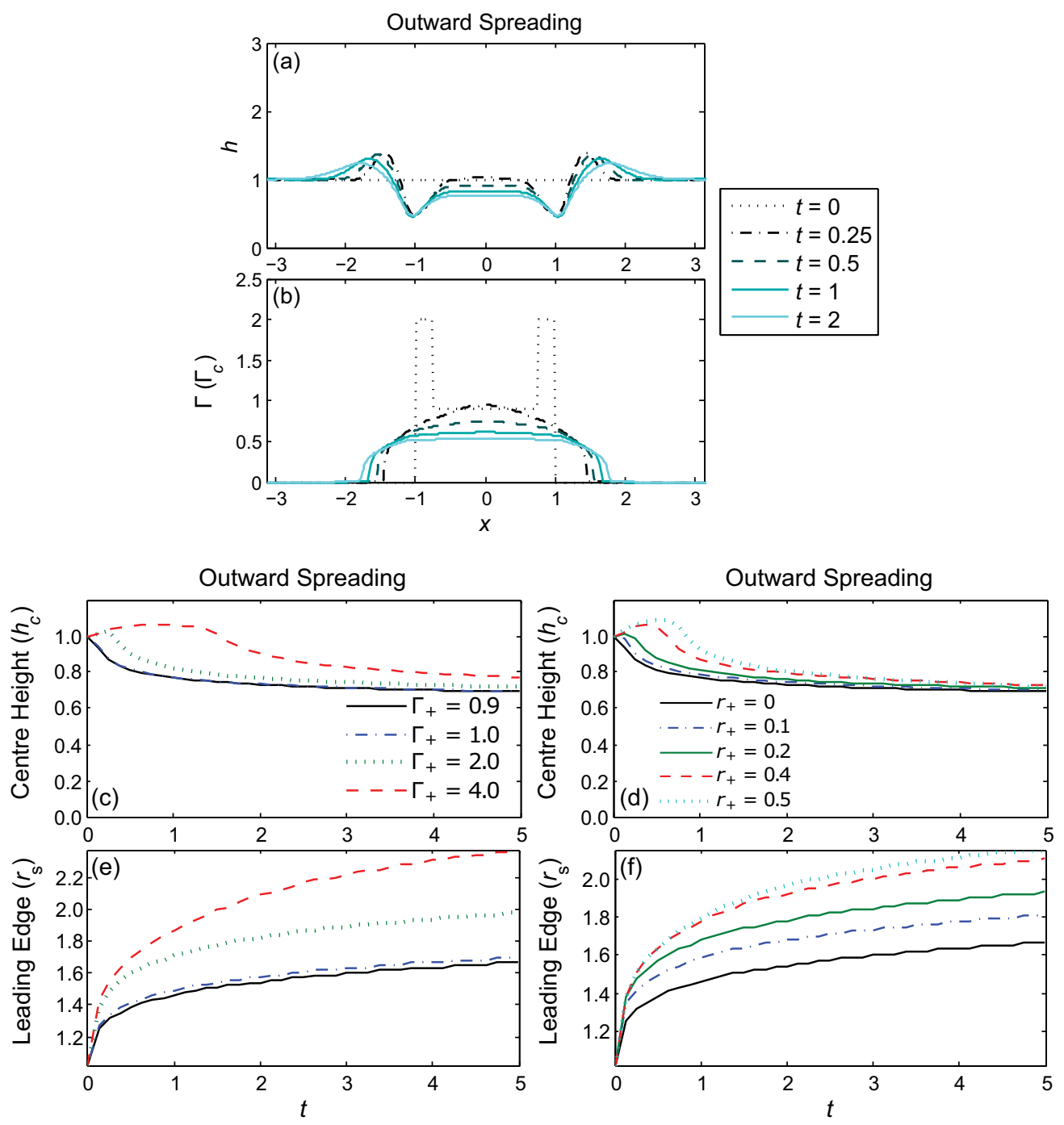

Figure 13. The dynamics of the central fluid height $h_{c}$ and the surfactant leading edge $r_{s}$ for outward spreading performed with an additional annulus of surfactant at ring location. Plots (a), (b) have standard parameters and initial condition (IC5). Plots (c), (e) have annulus width $r_{+}=0.25$ and vary $\Gamma_{+}$. Plots (d), (f) have fixed annulus surfactant concentration $\Gamma_{+}=2.0$ and vary $r_{+}$.

\section{Conclusion}

In this paper, we have tested ways in which the choice of a realistic EoS relating surface tension to surfactant concentration influences the outcome of numerical simulations. While simplified equations of state have dominated previous studies, including our own, such as $[2,3,5-7,13,20-22,27,28,36,44,45]$, we find that the spatiotemporal dynamics of surfactant spreading on a thin layer of viscous Newtonian fluid are in fact highly dependent on this choice. Therefore, it is important to incorporate empirical measurements of $\tilde{\sigma}(\tilde{\Gamma})$ 
for the specific materials under investigation. In particular, the correct choice allows for simulations to capture the detailed morphology of a spreading front of surfactant (Investigation 1) which have a distinctive reservoir if the initial surfactant concentration is above $\Gamma_{c}$.

We additionally observe that accurately measuring the correct value of $\tilde{\Gamma}_{c}$ in the EoS (or picking a different surfactant) will impact predictions for timescales. This effect arises because gradients in $\sigma$ are much stronger for intermediate values of surfactant concentration (above the transition point from the gas phase and below the critical concentration). The findings indicate that the use of a single spreading parameter $S$ is an oversimplification, and other choices should be explored. This is particularly important for outward spreading (as compared with inward spreading), but only in parameter regimes in which the gradient of $\sigma(\Gamma)$ is strongly affected.

Varying the small non-dimensional parameters $\kappa$ and $\delta$ does not largely affect the central fluid height evolution nor the surfactant leading edge location for inward or outward spreading. Varying $\beta$ does impact the dynamics, but the solutions can be scaled to show the self-similar behaviour in the system. The lack of sensitivity to $\beta, \kappa, \delta$ explains why prior attempts to resolve timescale issues by adjusting the non-dimensional parameters have failed. (Even so, as long as the correct values for the fluid are used in the model, there should be no flexibility to tune these parameters to better match model and experiment.) A different non-dimensionalization of the timescale could solve some issues with quantitatively predicting spreading rates. Future work could incorporate new experimental measurements of surface shear and dilational viscosities into the model as suggested in [17]. The effective surface viscosity may be much lower than that of the viscous fluid, which is the viscosity assumed in our model. This could motivate scaling factors to improve agreement with experimental dynamics.

Finally, the use of empirically relevant equations of state in simulations can help experimentalists address how their methods of creating repeatable initial conditions may be impacting results. We used the empirically correct EoS to test for possible artefacts from the use of a retaining ring to generate initial conditions. We find that the presence of a fluid meniscus created by such rings will not affect the dynamics. However, if the experimental apparatus also generates a region of surplus surfactant, this can generate long-lived concentration gradients near the original location of the ring, and also affect the velocity of the spreading front.

These findings will aid in providing future improvements in the quantitative agreements between simulations and experiments which have so far been elusive [40]. In addition, because simulations are more time-efficient to perform than laboratory experiments, improved agreement will aid experimentalists by providing a new tool for finding promising new regimes of behaviour.

\section{Acknowledgements}

We thank Stephen Strickland for sharing data from experiments, Jonathan Claridge and Jeffrey Wong for collaboration on the code, and Michael Shearer and Ellen Swanson for their contributions regarding the outward spreading system. We thank the referees for their helpful suggestions. 


\section{References}

[1] Avanti POLAR LIPIDS. WWw .avantilipids.com/index.php?option=com_content\&view= article\&id $=1028 \&$ Itemid $=220 \&$ catnumber $=810131$

[2] Angelini, T. E., Roper, M., Kolter, R., Weitz, D. A. \& Brenner, M. P. (2009) Bacillus subtilis spreads by surfing on waves of surfactant. Proc. Natl. Acad. Sci. 106(43), 18109-18113.

[3] Barrett, J. W., Garcke, H. \& NÜrnberG, R. (2003) Finite element approximation of surfactant spreading on a thin film. SIAM J. Numer. Anal. 41(4), 1427-1464.

[4] Borgas, M. S. \& Grotberg, J. B. (1988) Monolayer flow on a thin film. J. Fluid Mech. 193, 151-170.

[5] Braun, R. J. (2012) Dynamics of the tear film. Annu. Rev. Fluid Mech. 44, 267-297.

[6] Bull, J. \& Grotberg, J. (2003) Surfactant spreading on thin viscous films: Film thickness evolution and periodic wall stretch. Exp. Fluids 34(1), 1-15.

[7] Conti, C., Autry, E. A., Kronmiller, G. \& Levy, R. (2013) The effects of spatial and temporal grids on simulations of thin films with surfactant. SIURO 916, 81-93.

[8] Craster, R. \& Matar, O. (2000) Surfactant transport on mucus films. J. Fluid Mech. 425, 235-258.

[9] Craster, R. \& Matar, O. (2009) Dynamics and stability of thin liquid films. Rev. Mod. Phys. 81(3), 1131.

[10] De Wit, A., Gallez, D. \& Christov, C. (1994) Nonlinear evolution equations for thin liquid films with insoluble surfactants. Phys. Fluids (1994-present) 6(10), 3256-3266.

[11] Espinosa, F., Shapiro, A., Fredberg, J. \& Kamm, R. (1993) Spreading of exogenous surfactant in an airway. J. Appl. Phys. 75(5), 2028-2039.

[12] Fallest, D. W., Lichtenberger, A. M., Fox, C. J. \& Daniels, K. E. (July 2010) Fluorescent visualization of a spreading surfactant. New J. Physics 12(7), 73029.

[13] Garcke, H. \& Wieland, S. (2006) Surfactant spreading on thin viscous films: Nonnegative solutions of a coupled degenerate system. SIAM J. Math. Anal. 37(6), 2025-2048.

[14] Gaver, D. P. \& Grotberg, J. B. (1990) The dynamics of a localized surfactant on a thin film. J. Fluid Mech. 213, 127-148.

[15] Gaver, D. P. \& Grotberg, J. B. (1992) Droplet spreading on a thin viscous film. J. Fluid Mech. 235, 399-414.

[16] Halpern, D. \& Grotberg, J. (1993) Surfactant effects on fluid-elastic instabilities of liquidlined flexible tubes: A model of airway closure. J. Biomech. Eng. 115(3), 271-277.

[17] Heidari, A. H., Braun, R. J., Hirsa, A. H., Snow, S. A. \& Naire, S. (2002) Hydrodynamics of a bounded vertical film with nonlinear surface properties. J. Colloid Interface Sci. 253(2), 295-307.

[18] J. Claridge, R. Levy \& J. Wong https://github.com/claridge/implicit_solvers/blob/ master/ImplicitSolversPaper.pdf

[19] J. Claridge, R. Levy \& J. Wong https://github.com/claridge/implicit_solvers/

[20] Jensen, O. (1994) Self-similar, surfactant-driven flows. Phys. Fluids (1994-present) 6(3), 10841094.

[21] Jensen, O. \& Grotberg, J. (1992) Insoluble surfactant spreading on a thin viscous film: Shock evolution and film rupture. J. Fluid Mech. 240, 259-288.

[22] Jensen, O. \& Grotberg, J. (1993) The spreading of heat or soluble surfactant along a thin liquid film. Phys. Fluids A: Fluid Dyn. (1989-1993) 5(1), 58-68.

[23] Kaganer, V., Möhwald, H. \& Dutta, P. (April 1999) Structure and phase transitions in Langmuir monolayers. Rev. Mod. Phys. 71(3), 779-819.

[24] Karsa, D. (1999) Industrial Applications of Surfactants IV. Special Publication/Royal Society of Chemistry. Vol. 230, Elsevier Science.

[25] Levy, R. (2005) Partial differential equations of thin liquid films: Analysis and numerical simulation, thesis, North Carolina State University.

[26] Levy, R., Hill, D. B., Forest, M. G. \& Grotberg, J. B. (2014) Pulmonary fluid flow challenges for experimental and mathematical modeling. Integrative Comparative Biol. 54(6), 985-1000. 
[27] Levy, R. \& Shearer, M. (2006) The motion of a thin liquid film driven by surfactant and gravity. SIAM J. Appl. Math. 66(5), 1588-1609.

[28] Levy, R., Shearer, M. \& Witelski, T. P. (2007) Gravity-driven thin liquid films with insoluble surfactant: Smooth traveling waves. Eur. J. Appl. Math. 18(06), 679-708.

[29] Naire, S., Braun, R. J. \& Snow, S. A. (2004) A 2+1 dimensional insoluble surfactant model for a vertical draining free film. J. Comput. Appl. Math. 166(2), 385-410.

[30] Oron, A., Davis, S. H. \& Bankoff, S. G. (1997) Long-scale evolution of thin liquid films. Rev. Mod. Phys. 69(3), 931.

[31] Otis, D., Johnson, M., Pedley, T. \& Kamm, R. (1993) Role of pulmonary surfactant in airway closure: A computational study. J. Appl. Physiology 75(3), 1323-1333.

[32] Pereira, A., Trevelyan, P., Thiele, U. \& Kalliadasis, S. (2007) Dynamics of a horizontal thin liquid film in the presence of reactive surfactants. Phys. Fluids (1994-present) 19(11), 112102.

[33] Peterson, E. R. (2010) Flow of thin liquid films with surfactant: Analysis, numerics, and experiment.

[34] Peterson, E. R. \& Shearer, M. (2011) Radial spreading of a surfactant on a thin liquid film. Appl. Math. Res. Express 2011(1), 1-22.

[35] Reis, P., Holmberg, K., Watzke, H., Leser, M. E. \& Miller, R. (2009) Lipases at interfaces: A review. Adv. Colloid Interface Sci. 147-148, 237-250.

[36] Renardy, M. (1996) A singularly perturbed problem related to surfactant spreading on thin films. Nonlinear Anal.: Theory, Methods Appl. 27(3) (1996), 287-296.

[37] Shrive J. D. A., Brennan J. D., Brown R. S. \& Krull U. J. (1995) Optimization of selfquenching response of nitrobenzoxadiazole dipalmitoylphosphatidylethanolaminein phospholipid membranes for biosensor development. Appl. Spectrosc. 49, 304-313.

[38] STRICKLAND, S. L. Surfactant dynamics: Spreading and wave induced dynamics of a monolayer.

[39] Strickland, S. L., Hin, M., Sayanagi, M. R., Gaebler, C., Daniels, K. E., Levy, R. \& Conti, C. (April 2014) Self-healing dynamics of surfactant coatings on thin viscous films. Phys. Fluids 26(4), 042109.

[40] Swanson, E. R., Strickland, S. L., Shearer, M. \& Daniels, K. E. (2015) Surfactant spreading on a thin liquid film: Reconciling models and experiments. J. Eng. Math. 94, 63-79.

[41] Tiberg, F. \& Cazabat, A.-M. (1994) Spreading of thin films of ordered nonionic surfactants - Origin of the stepped shape of the spreading precursor. Langmuir 10(7), 2301-2306.

[42] Troian, S. M., Herbolzheimer, E., Safran, S. A., Joanny, J. F., Wu, X. L. \& Safran, S. A. (1989) Fingering instability in thin wetting films. Phys. Rev. Lett. 62, 1496-1499.

[43] Troian, S. M., Herbolzheimer, E. \& Safran, S. A. (1990) Model for the fingering instability of spreading surfactant drops. Phys. Rev. Lett. 65, 333-336.

[44] Warner, M., Craster, R. \& Matar, O. (2004) Fingering phenomena associated with insoluble surfactant spreading on thin liquid films. J. Fluid Mech. 510, 169-200.

[45] Witelski, T. P., Shearer, M. \& Levy, R. (2006) Growing surfactant waves in thin liquid films driven by gravity. Appl. Math. Res. Express 2006, 15487. 